\title{
Observation of the Self-Modulation Instability via Time-Resolved Measurements
}

\author{
M. Gross, ${ }^{1,}{ }^{*}$ J. Engel, ${ }^{1}$ J. Good,${ }^{1}$ H. Huck, ${ }^{1}$ I. Isaev, ${ }^{1}$ G. Koss, ${ }^{1}$ M. Krasilnikov, ${ }^{1}$ O. Lishilin, ${ }^{1}$ G. Loisch,${ }^{1}$ \\ Y. Renier, ${ }^{1}$ T. Rublack, ${ }^{1}$ F. Stephan, ${ }^{1}$ R. Brinkmann, ${ }^{2}$ A. Martinez de la Ossa, ${ }^{2,3}$ J. Osterhoff, ${ }^{2}$ D. Malyutin, ${ }^{4}$ \\ D. Richter, ${ }^{4}$ T. Mehrling, ${ }^{3,5}$ M. Khojoyan, ${ }^{6}$ C. B. Schroeder, ${ }^{7}$ and F. Grüner ${ }^{3,8}$ \\ ${ }^{1}$ Deutsches Elektronen-Synchrotron DESY, Platanenallee 6, 15738 Zeuthen, Germany \\ ${ }^{2}$ Deutsches Elektronen-Synchrotron DESY, Notkestr. 85, 22607 Hamburg, Germany \\ ${ }^{3}$ Universität Hamburg, Luruper Chaussee 149, 22761 Hamburg, Germany \\ ${ }^{4}$ Helmholtz-Zentrum Berlin für Materialien \& Energie, Albert-Einstein-Strasse 15, 12489 Berlin, Germany \\ ${ }^{5}$ Instituto Superior Técnico, Avenida Rovisco Pais 1, 1049-001 Lisbon, Portugal \\ ${ }^{6}$ LLR (Laboratoire Leprince-Ringuet), CNRS and Ecole Polytechnique, Palaiseau UMR7638, France \\ ${ }^{7}$ Lawrence Berkeley National Laboratory, 1 Cyclotron Road, Berkeley, California 94720, USA \\ ${ }^{8}$ Center for Free-Electron Laser Science, Notkestrasse 85, 22607 Hamburg, Germany
}

(Received 30 October 2017; published 6 April 2018)

\begin{abstract}
Self-modulation of an electron beam in a plasma has been observed. The propagation of a long (several plasma wavelengths) electron bunch in an overdense plasma resulted in the production of multiple bunches via the self-modulation instability. Using a combination of a radio-frequency deflector and a dipole spectrometer, the time and energy structure of the self-modulated beam was measured. The longitudinal phase space measurement showed the modulation of a long electron bunch into three bunches with an approximately $200 \mathrm{keV} / c$ amplitude momentum modulation. Demonstrating this effect is a breakthrough for proton-driven plasma accelerator schemes aiming to utilize the same physical effect.
\end{abstract}

DOI: $10.1103 /$ PhysRevLett.120.144802

Plasma acceleration has the potential to dramatically miniaturize accelerators for modern applications such as $x$-ray free electron lasers or high-energy physics, owing to the extremely high acceleration fields, of the order of $1-100 \mathrm{GV} / \mathrm{m}$, that can be obtained in a plasma. These large fields may be driven by an intense laser pulse [1] or a high-energy particle bunch [2]. Proton bunches as drivers are intriguing since, due to the large proton mass, these bunches can easily store a large amount of energy. In principle, a high-energy proton beam could accelerate electrons to $\mathrm{TeV}$ energies in a single plasma acceleration stage [3]. Proton bunches with the necessary energies are already available from conventional accelerators such as the Large Hadron Collider at CERN (European Centre for Particle Physics). However, to excite a plasma wakefield efficiently the length of the proton bunch should be shorter than the plasma wavelength. To reach acceleration gradients of $1 \mathrm{GV} / \mathrm{m}$ - an order of magnitude higher than conventional radio frequency (rf) technology - the plasma density needs to be of the order of $n_{p} \sim 10^{15} \mathrm{~cm}^{-3}$, which

Published by the American Physical Society under the terms of the Creative Commons Attribution 4.0 International license. Further distribution of this work must maintain attribution to the author(s) and the published article's title, journal citation, and DOI. translates to a plasma wavelength of $\sim 1 \mathrm{~mm}$, but available proton bunches have lengths of about $\sim 10 \mathrm{~cm}$.

To solve this problem it was suggested [4] to split up the bunch into sub-bunches with lengths of the plasma period by utilizing the self-modulation instability in a plasma [5-7]. The modulated bunch can then efficiently drive a large plasma wakefield for acceleration. Although an experimental demonstration of the self-modulation instability was lacking, an experiment by the Advanced Wakefield Experiment (AWAKE) collaboration to demonstrate proton-driven plasma wakefield acceleration at CERN was initiated [8,9]. The physics of the linear selfmodulation instability using lepton beams is analogous to proton beams [10], and scaled experiments to demonstrate the instability were attempted using electron beams [11,12], with indirect indications of beam modulation.

In this Letter, we present direct time-resolved measurements of an electron beam modulated via the selfmodulation instability in a plasma. These experiments were performed at the Deutsches Elektronen Synchrotron (DESY), specifically the Photo Injector Test facility at DESY in Zeuthen (PITZ). PITZ operates an electron linear accelerator producing brilliant electron bunches with final energies of up to $25 \mathrm{MeV}[13,14]$. Using electrons has the advantage that due to their much lower mass the selfmodulation instability may be observed in only centimeters of plasma [10], compared to meters needed for protons $[8,9]$. These longitudinal phase space measurements 
unambiguously demonstrate the self-modulation of an electron bunch, modulating a $22.3 \mathrm{MeV}$ electron bunch into three bunchlets with a $\sim 200 \mathrm{keV} / c$ amplitude momentum modulation.

The beam self-modulation experiment uses several key components at PITZ. The first item is the photocathode laser, which is used to generate the electron bunches. This laser includes a pulse shaper [15] to generate almost arbitrary longitudinal laser pulse shapes with a FWHM length of up to $25 \mathrm{ps}$. Temporal flat top shapes with rise and fall times as short as 2 ps can be generated [14]. This is an important item since it is necessary to seed a plasma wake by a sharp rise of the electron density at the head of the bunch [16]. (In case of the AWAKE experiment the sharp rise is implemented by a laser-induced copropagating ionization front, resulting in a step of the plasma density [8].) The other main item is the system to measure the longitudinal phase space of the electron bunches after interaction with the plasma. This consists of two devices: a rf deflector [17,18] and a dipole spectrometer [19], which work on orthogonal axes to separate the bunch electrons by longitudinal position and momentum. This generates an instantaneous picture of the longitudinal phase space on a cerium doped yttrium aluminum garnet (Ce:YAG) scintillator screen in the dispersive section downstream of the dipole. A plasma cell was developed for the experiments to generate a plasma column with a density and length in electron beam direction as determined from simulations [20]: The electron density of the plasma channel should be $n_{p} \sim 10^{14}-10^{15} \mathrm{~cm}^{-3}$, which is equivalent to a plasma wavelength of $\sim 1-3 \mathrm{~mm}$. Our electron bunches are about $6 \mathrm{~mm}$ long (equivalent to a duration of $20 \mathrm{ps}$ ) and with the self-modulation period equaling the plasma wavelength [4] two to six modulation periods are expected, promising good visibility. The modulation depth increases for a propagation distance up to $l \sim 6-8 \mathrm{~cm} \mathrm{[21].} \mathrm{Afterwards} \mathrm{the} \mathrm{self-modu-}$ lation saturates and the bunch quality slowly deteriorates due to dephasing and competing plasma effects, e.g., hosing $[22,23]$. Therefore, we used a plasma channel length of $8 \mathrm{~cm}$ in the experiments, resulting in a self-modulation signal with maximal achievable amplitude and minimal distortions. The heat pipe oven technology, capable of generating plasma with a density of $10^{14}-10^{15} \mathrm{~cm}^{-3}$ for beam driven plasma acceleration experiments [24] was utilized. For our purposes we developed and built a heat pipe oven that couples a pulsed UV laser through side windows to ionize lithium vapor [25]. This design allows flexible generation of the plasma channel by generating arbitrary transverse laser intensity profiles. The length of the plasma channel in the electron beam direction is defined by the horizontal width of the ionization laser beam. A schematic of the overall experimental setup is shown in Fig. 1.

The electron bunches were prepared as a flat top longitudinally with rise and fall times of $\sim 2$ ps [14]. The $22.3 \mathrm{MeV}$ beam had a rms momentum spread of

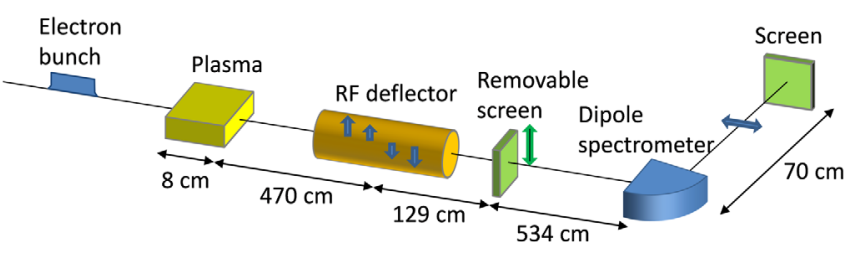

FIG. 1. Schematic of the experimental setup. After passing through the plasma the electron bunch enters a diagnostics section. The bunch is streaked by a rf deflector vertically and its longitudinal charge distribution can then be observed on a removable Ce: YAG scintillator screen. Alternatively, the bunch can be further transported to a dipole spectrometer where the bunch is energy dispersed horizontally. This allows one to observe the longitudinal phase space of the bunch on a second Ce:YAG screen.

$0.1 \mathrm{MeV} / c$ and a bunch charge of $970 \mathrm{pC}$. The electron beam was strongly focused into the plasma to maximize the amplitude of the induced plasma wakefield, and hence, the self-modulation depth. A combination of a focusing solenoid, positioned at the electron gun and four quadrupole magnets in front of the plasma cell focused the beam to a transverse rms size of $\sim 100 \mu \mathrm{m}$ [25] at the entrance of the plasma. The plasma cell was operated with a heater temperature of $700^{\circ} \mathrm{C}$ and an argon buffer gas pressure of 1.4 mbar. UV absorption measurements done before inserting the plasma cell into the accelerator beam line yielded a lithium vapor density of $4 \times 10^{15} \mathrm{~cm}^{-3}$ for these conditions. The ionization laser delivered pulses with an energy of up to $150 \mathrm{~mJ}$ to the plasma cell. This photoionization method leads to incomplete ionization of the lithium vapor, so that the plasma density is about 1 order of magnitude lower than the vapor density $[20,25]$. The plasma densities given below were deduced from the measured plasma wavelength.

Figure 2 shows an electron bunch, which was resolved in time with the rf deflector as described above. The bunch was recorded on the removable Ce:YAG screen downstream of the rf deflector as a comparison between (a) plasma off and (b) maximal plasma density. The head of the electron bunch is on the right side in Figs. 2(a) and 2(b). In the case without plasma the longitudinal flat top shape with a FWHM length of $24 \mathrm{ps}$ and a rise time of $2 \mathrm{ps}$ is measured. This compares well with measurements of the laser pulse rise time. When the bunch is passing through the plasma, the effect of the selfmodulation can be seen: The head of the electron bunch is slightly compressed for the first $1.3 \mathrm{ps}$. Behind the head is a region of strong defocusing with a nearly sinusoidal envelope followed by another focusing region. The distance between the bunch head and this focusing region is one plasma period, which can be evaluated from Fig. 2(b) as $\sim 10$ ps. This corresponds to a plasma wavelength of $\sim 3 \mathrm{~mm}$ and a plasma density of $\sim 10^{14} \mathrm{~cm}^{-3}$. Behind the focusing region near the middle of the bunch another defocusing region is visible marking a second period of the plasma wake.

For the measurement of the longitudinal phase space we used electron bunches with the same parameters as above. 


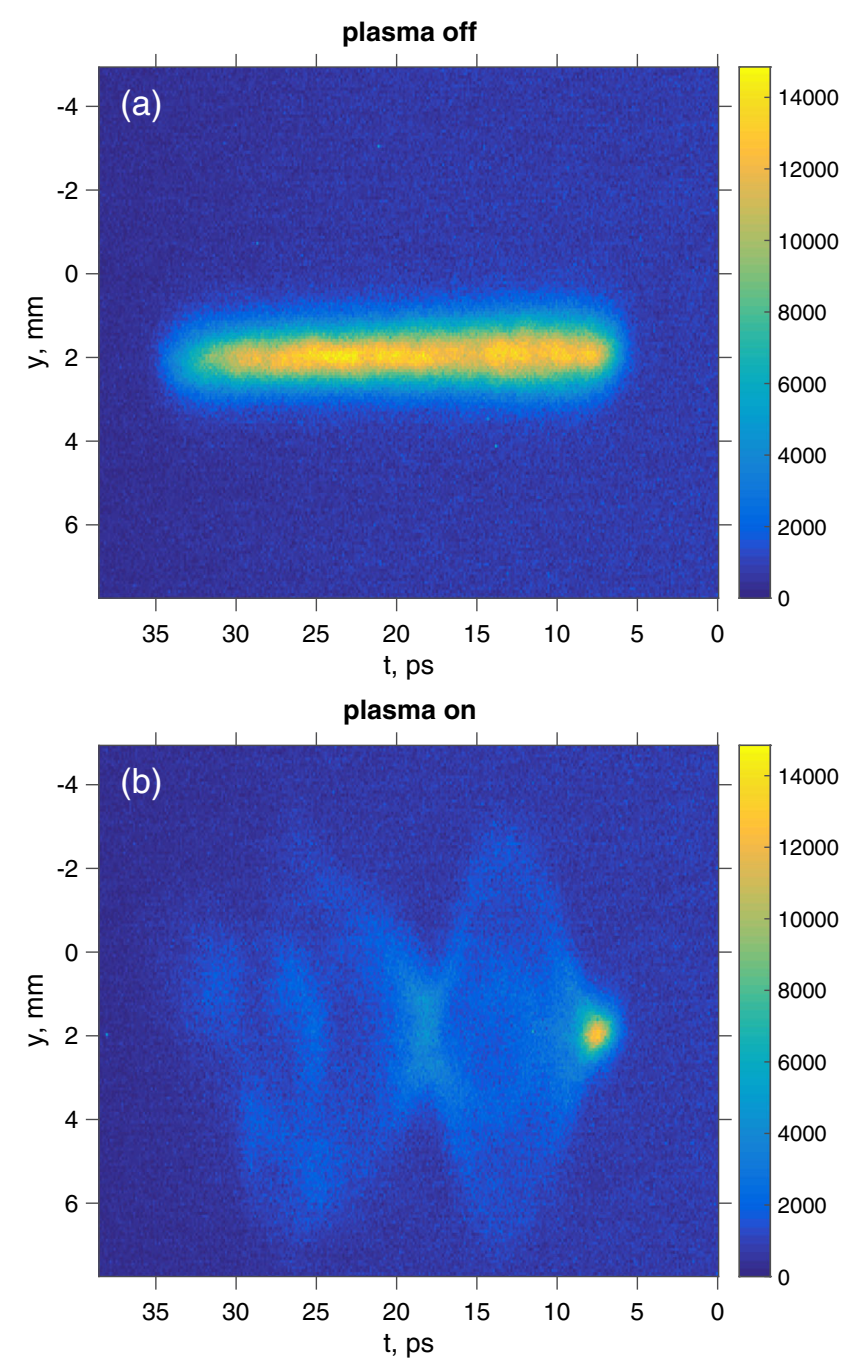

FIG. 2. Measured time-resolved electron bunch - the head of the bunch is on the right side; the color bar indicates measured intensity up to the maximal output value of the observing camera. Examples of bunches streaked with the rf deflector after passing the plasma cell with (a) a pure lithium vapor and (b) $8 \mathrm{~cm}$ of plasma with a density of $\sim 10^{14} \mathrm{~cm}^{-3}$. Without plasma the longitudinal bunch shape is a flat top, as generated in the accelerator. When the bunch is passing through the plasma it is self-modulated at the plasma period of $\sim 10 \mathrm{ps}$, corresponding to a plasma wavelength of $\sim 3 \mathrm{~mm}$. Two modulation periods are visible.

The heater temperature of the plasma cell was increased to $710^{\circ} \mathrm{C}$, resulting in a slightly higher lithium vapor density of $5 \times 10^{15} \mathrm{~cm}^{-3}$. Figure 3 shows the longitudinal phase space of the electron bunches, again as a comparison between (a) plasma off and (b) maximal plasma density. The head of the electron bunch is on the right side in Figs. 3(a) and 3(b). The longitudinal phase space of the bunch without plasma interaction is nearly linear. The electron bunch passing through the plasma shows clear signatures of self-modulation. First, the bunch is focused and defocused alternately with the period of the plasma wavelength, caused by the transverse electric fields in the plasma wake. Second, the corresponding
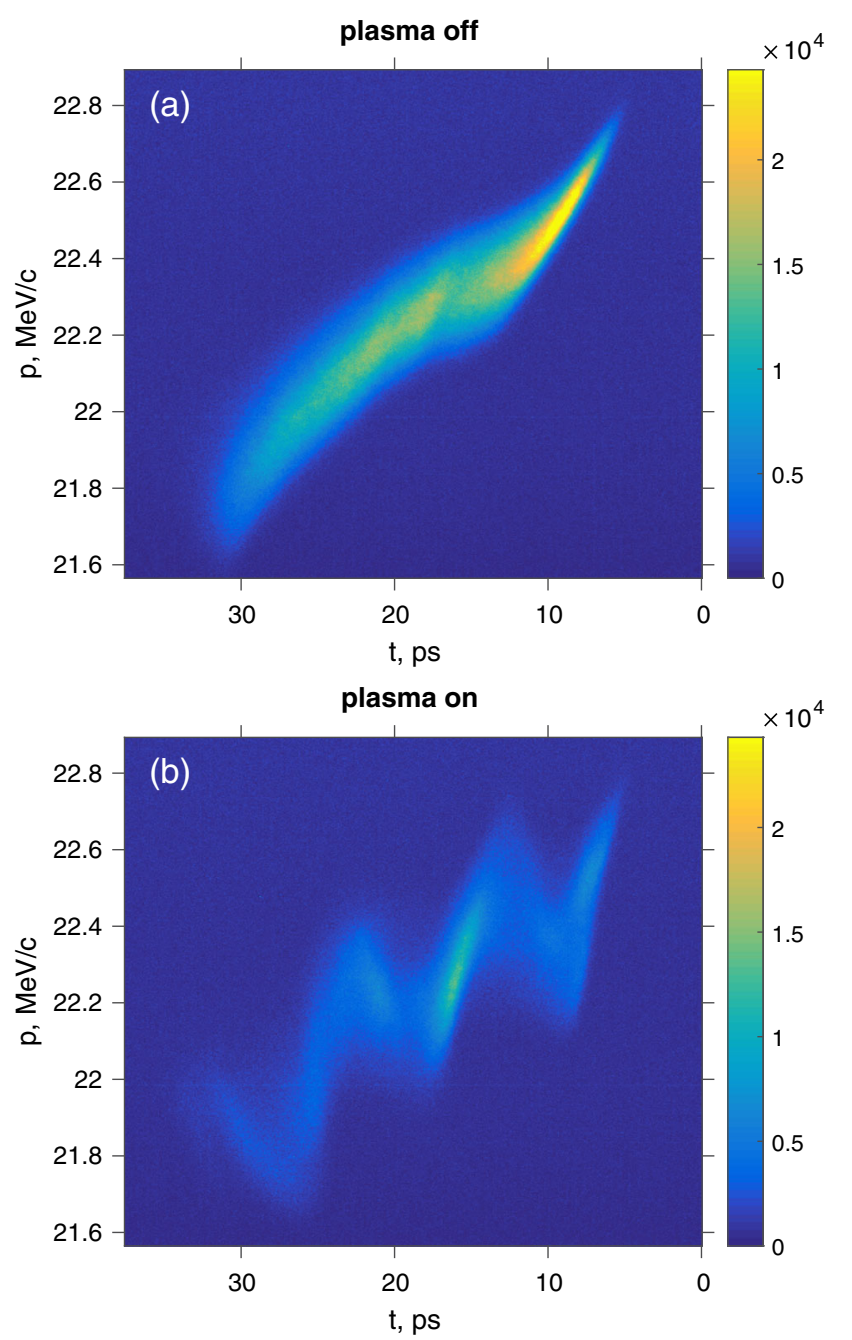

FIG. 3. Measured longitudinal phase space of an electron bunch - the head of the bunch is on the right side; the color bar indicates measured intensity up to the maximal output value of the observing camera. Examples of bunches streaked with the rf deflector and energy dispersed with the dipole after passing through the plasma cell with (a) a pure lithium vapor and (b) $8 \mathrm{~cm}$ of plasma with a density of slightly higher than $10^{14} \mathrm{~cm}^{-3}$. Without plasma the longitudinal phase space is nearly linear. When the bunch is passing through the plasma it shows evidence of self-modulation: transverse electric fields in the plasma wake are causing alternate focusing and defocusing, while the corresponding longitudinal electric fields lead to a momentum modulation. Three modulation periods are visible-one more than in Fig. 2 due to the higher plasma density in this case.

longitudinal electric fields lead to a momentum modulation, with an amplitude of $\sim 200 \mathrm{keV} / c$.

Here the ratio of beam density to plasma density is $n_{b} / n_{p}=0.2$ (i.e., overdense beam-plasma interaction), and the normalized charge per unit length, which determines the normalized amplitude of the accelerating field [26], is $\left(n_{b} / n_{p}\right)\left(k_{p} \sigma_{r}\right)^{2}=0.01$, where $\sigma_{r}$ is the transverse beam size and $k_{p}$ the plasma wave number. Hence the excited wakefield is initially in the linear regime. Since the 
bunches are long compared to the plasma wavelength, selfmodulation occurs, seeded by the fast rise of the head of the bunch. The seed wakefield grows quadratically with respect to the propagation distance and triggers the self-modulation instability [16]. The self-modulation instability results in an exponential growth of the wakefield amplitude, $\exp (N)$, with the number of $e$-foldings,

$$
N=\left(3^{3 / 2} / 4\right)\left[\left(n_{b} / n_{p}\right)\left(m_{e} / M_{b}\right) k_{p}^{3} L_{b} l^{2} / \gamma\right]^{1 / 3},
$$

where $L_{b}$ is the bunch length, $l$ is the propagation distance, $\gamma$ the Lorentz factor of the beam energy, $\left(m_{e} / M_{b}\right)$ the ratio of electron mass to the mass of the beam particles (electrons or protons), and $k_{p} \sigma_{r}<1$ [6]. For the experimental parameters, the number of $e$-foldings $N$ of the instability growth at the tail of the bunch is $N=16$. Hence, we expect the bunch modulation to enter a saturated regime. This evaluation is supported by numerical simulation of the experimental parameters. An electron bunch with a charge distribution as measured in our experiment was implemented in ASTRA [27] and transported to the entry of the plasma cell. This particle distribution was then imported into the particle-in-cell code HiPACE [28] for simulation of the bunch-plasma interaction. Results for three different gun solenoid focusing currents that are close to the one used in the experiment (382A) are depicted in Fig. 4. The simulated transverse electric field, which is the cause of the self-modulation, shows rapid growth, followed by saturation.

Note that the dependence of self-modulation instability properties on beam parameters, especially beam emittance, was studied for a number of experimental setups to find limitations for self-modulation instability growth. It was found that the PITZ setup is best suited to avoid emittance driven erosion [29].

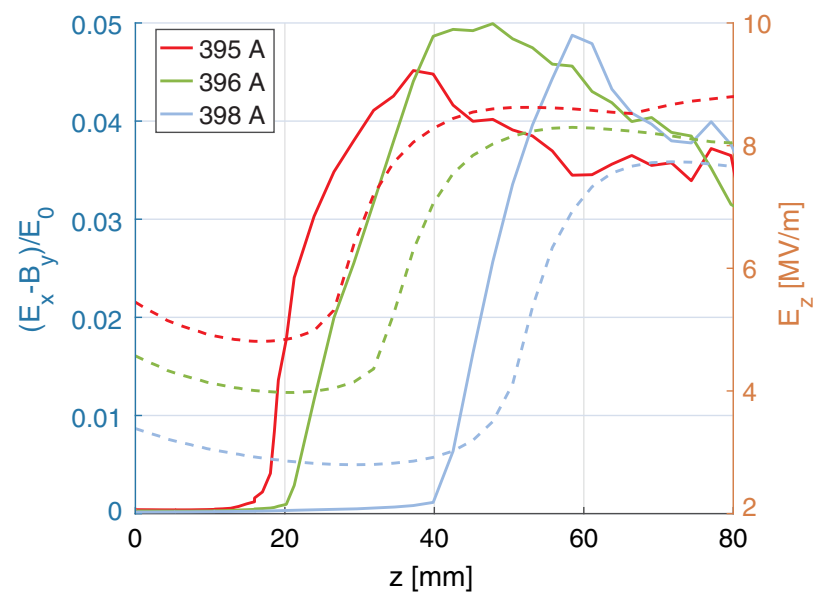

FIG. 4. Simulated electromagnetic fields along the plasma channel with electron bunch and plasma conditions as in the experiment. Shown are maximum transverse wakefields along the simulation box (solid lines, left axis) and the longitudinal electric field of the first modulation maximum (dashed lines, right axis).
Figure 2(b) shows that the self-modulation instability is the source of the beam modulation: the bunch radius is enlarged significantly around the phase of maximal defocusing. This would not be expected without influence from the self-modulation instability since the initial transverse forces generated by the bunch are focusing at all phases, an effect well known as plasma lensing in an overdense plasma [30]. Since the initial bunch radius $(0.1 \mathrm{~mm})$ is larger than the matched radius in the plasma $(0.05 \mathrm{~mm})$, one expects the bunch radius at any point along the bunch to be less than the initial radius as it undergoes betatron motion. The fact that Fig. 2(b) shows a bunch radius larger than $0.1 \mathrm{~mm}$ is evidence that the wakefield amplitude grew sufficiently large (beyond the seed wakefield), introducing a feedback between the wake and bunch distribution to generate regions of strong defocusing.

While the head of the bunch is tightly focused after passing the plasma, the focusing region one plasma wavelength behind has a much larger diameter, as can be seen in Fig. 2(b). An explanation for the larger diameter is phase slippage between electron bunch and wakefield; i.e., the bunch behind the head slips through the focusing and defocusing regions. If the modulation process is caused by the self-modulation instability the velocity of the resulting wakefield is less than the driver velocity [6]. For the experimental parameters the Lorentz factor of the wake at the first focusing region behind the bunch head can be calculated to be $\gamma_{\text {wake }}=2.5$. This is much smaller than the Lorentz factor of the beam energy $(\gamma=44.6)$, leading to slippage between bunch and wakefield. The phase of the wakefield is retarded by $\pi$ after a propagation distance of $\sim 2 \mathrm{~cm}$, preventing tight focusing. The head of the bunch does not experience slippage of the wakefield, which is consistent with the observations shown in Fig. 2(b). These experimental observations are consistent with exponential self-modulation growth as expected from the self-modulation instability.

The experimental results presented here are direct evidence of the self-modulation of a long (with respect to the plasma wavelength) electron bunch in a plasma. An rf deflector was utilized to characterize the temporal structure of a self-modulated electron bunch, showing the transverse modulation. By including a dipole spectrometer we were also able to demonstrate the momentum modulation of the bunch. The experimental evidence confirms the selfmodulation instability as cause for the observed modulation. Simulations based on the experimental conditions support this conclusion. Further experiments with reduced plasma density have shown the expected increase of the observed self-modulation period for both classes of timeresolved experiments: versus transverse bunch size and versus momentum. Future experiments are planned to investigate the influence of plasma inhomogeneity (modulation, ramps, and noise) on the self-modulation. This demonstration experiment is an important step on the road 
to develop plasma wakefield acceleration into the next generation of useable accelerator technology.

The authors thank Sebastian Philipp and Anne Oppelt for strong support to set up the experimental apparatus. We thank all active members of the PITZ collaboration, especially Galina Asova and Prach Boonpornprasert for their experimental assistance. We acknowledge Patric Muggli and Erdem Öz for helpful discussions on the aspects of the plasma cell design. Bernhard Hidding contributed financially to the experimental setup. Work was supported by the German federal ministry of education and research, Grant No. 05H12GU6 and by the Director, Office of Science, Office of High Energy Physics, of the U.S. Department of Energy under Contract No. DE-AC02-05CH11231.

*matthias.gross@desy.de

[1] E. Esarey, C. B. Schroeder, and W. P. Leemans, Physics of laser-driven plasma-based electron accelerators, Rev. Mod. Phys. 81, 1229 (2009).

[2] I. Blumenfeld et al., Energy doubling of $42 \mathrm{GeV}$ electrons in a metre-scale plasma wakefield accelerator, Nature (London) 445, 741 (2007).

[3] A. Caldwell, K. Lotov, A. Pukhov, and F. Simon, Proton-driven plasma-wakefield acceleration, Nat. Phys. 5, 363 (2009).

[4] N. Kumar, A. Pukhov, and K. Lotov, Self-Modulation Instability of a Long Proton Bunch in Plasmas, Phys. Rev. Lett. 104, 255003 (2010).

[5] A. Pukhov, N. Kumar, T. Tückmantel, A. Upadhyay, K. Lotov, P. Muggli, V. Khudik, C. Siemon, and G. Shvets, Phase Velocity and Particle Injection in a Self-Modulated Proton-Driven Plasma Wakefield Accelerator, Phys. Rev. Lett. 107, 145003 (2011).

[6] C. B. Schroeder, C. Benedetti, E. Esarey, F. J. Grüner, and W. P. Leemans, Growth and Phase Velocity of SelfModulated Beam-Driven Plasma Waves, Phys. Rev. Lett. 107, 145002 (2011).

[7] K. Lotov, Controlled self-modulation of high energy beams in a plasma, Phys. Plasmas 18, 024501 (2011).

[8] R. Assmann et al., Proton-driven plasma wakefield acceleration: a path to the future of high-energy particle physics, Plasma Phys. Controlled Fusion 56, 084013 (2014).

[9] E. Gschwendtner et al., AWAKE, The advanced proton driven plasma wakefield acceleration experiment at CERN, Nucl. Instrum. Methods Phys. Res., Sect. A 829, 76 (2016).

[10] J. Vieira, Y. Fang, W. B. Mori, L. O. Silva, and P. Muggli, Transverse self-modulation of ultra-relativistic lepton beams in the plasma wakefield accelerator, Phys. Plasmas 19, 063105 (2012).

[11] Y. Fang, V. E. Yakimenko, M. Babzien, M. Fedurin, K. P. Kusche, R. Malone, J. Vieira, W. B. Mori, and P. Muggli, Seeding of Self-Modulation Instability of a Long Electron Bunch in a Plasma, Phys. Rev. Lett. 112, 045001 (2014).

[12] E. Adli et al., Progress of plasma wakefield self-modulation experiments at FACET, Nucl. Instrum. Methods Phys. Res., Sect. A 829, 334 (2016).

[13] F. Stephan et al., Detailed characterization of electron sources yielding first demonstration of European X-ray
Free-Electron Laser beam quality, Phys. Rev. ST Accel. Beams 13, 020704 (2010).

[14] M. Krasilnikov et al., Experimentally minimized beam emittance from an L-band photoinjector, Phys. Rev. ST Accel. Beams 15, 100701 (2012).

[15] I. Will and G. Klemz, Generation of flat-top picosecond pulses by coherent pulse stacking in a multicrystal birefringent filter, Opt. Express 16, 14922 (2008).

[16] C. B. Schroeder, C. Benedetti, E. Esarey, F. J. Grüner, and W. P. Leemans, Coherent seeding of self-modulated plasma wakefield accelerators, Phys. Plasmas 20, 056704 (2013).

[17] L. Kravchuk et al., Layout of the PITZ transverse deflecting system for longitudinal phase space and slice emittance measurements, in Proceedings of LINAC (Tsukuba, Japan, 2010), p. 416.

[18] H. Huck et al., First results of commissioning of the PITZ transverse deflecting structure, JACoW, http://dx.doi.org/10 .18429/JACoW-FEL2015-MOP039, 2015.

[19] S. Rimjaem, J. W. Bähr, Y. Ivanisenko, M. Krasilnikov, J. Rönsch, F. Stephan, M. Joré, and A. Variola, Physics and technical design for the second high energy dispersive section at PITZ, in Proceedings of DIPAC (Basel, Switzerland, 2009), p. 107.

[20] M. Gross, R. Brinkmann, J. D. Good, F. Grüner, M. Khojoyan, A. Martinez de la Ossa, J. Osterhoff, G. Pathak, C. Schroeder, and F. Stephan, Preparations for a plasma wakefield acceleration (PWA) experiment at PITZ, Nucl. Instrum. Methods Phys. Res., Sect. A 740, 74 (2014).

[21] A. Martinez de la Ossa, M. Gross, F. Grüner, M. Khojoyan, M. Krasilnikov, A. Oppelt, F. Stephan, C. B. Schroeder, and J. Osterhoff, Self-modulation of long electron beams in plasma at PITZ, AIP Conf. Proc. 1507, 588 (2012).

[22] J. Vieira, W. B. Mori, and P. Muggli, Hosing Instability Suppression in Self-Modulated Plasma Wakefields, Phys. Rev. Lett. 112, 205001 (2014).

[23] C. B. Schroeder, C. Benedetti, E. Esarey, F. J. Grüner, and W. P. Leemans, Coupled beam hose and self-modulation instabilities in overdense plasma, Phys. Rev. E 86, 026402 (2012).

[24] P. Muggli, K. A. Marsh, S. Wang, C. E. Clayton, S. Lee, T. C. Katsouleas, and C. Joshi, Photo-ionized lithium source for plasma accelerator applications, IEEE Trans. Plasma Sci. 27, 791 (1999).

[25] O. Lishilin et al., First results of the plasma wakefield acceleration experiment at PITZ, Nucl. Instrum. Methods Phys. Res., Sect. A 829, 37 (2016).

[26] W. Lu, C. Huang, M. M. Zhou, W. B. Mori, and T. Katsouleas, Limits of linear plasma wakefield theory for electron or positron beams, Phys. Plasmas 12, 063101 (2005).

[27] K. Flöttmann, ASTRA particle tracking code, http://www .desy.de/ mpyflo/.

[28] T. Mehrling, C. Benedetti, C. B. Schroeder, and J. Osterhoff, HiPACE: a quasi-static particle-in-cell code, Plasma Phys. Controlled Fusion 56, 084012 (2014).

[29] K. Lotov, Effect of beam emittance on self-modulation of long beams in plasma wakefield accelerators, Phys. Plasmas 22, 123107 (2015).

[30] J. J. Su, T. Katsouleas, J. M. Dawson, and R. Fedele, Plasma lenses for focusing particle beams, Phys. Rev. A 41, 3321 (1990). 